\title{
Gain and noise properties of small-signal erbium-doped fiber amplifiers pumped in the
} 980-nm band

Pedersen, B.; Chirravuri, J.; Miniscalco, W. J.

Published in:

I E E E Photonics Technology Letters

Link to article, DOI:

10.1109/68.141966

Publication date:

1992

Document Version

Publisher's PDF, also known as Version of record

Link back to DTU Orbit

Citation (APA):

Pedersen, B., Chirravuri, J., \& Miniscalco, W. J. (1992). Gain and noise properties of small-signal erbium-doped fiber amplifiers pumped in the 980-nm band. I E E E Photonics Technology Letters, 4(6), 556-558.

https://doi.org/10.1109/68.141966

\section{General rights}

Copyright and moral rights for the publications made accessible in the public portal are retained by the authors and/or other copyright owners and it is a condition of accessing publications that users recognise and abide by the legal requirements associated with these rights.

- Users may download and print one copy of any publication from the public portal for the purpose of private study or research.

- You may not further distribute the material or use it for any profit-making activity or commercial gain

- You may freely distribute the URL identifying the publication in the public portal 


\title{
Gain and Noise Properties of Small-Signal Erbium-Doped Fiber Amplifiers Pumped in the $980-n m$ Band
}

\author{
B. Pedersen, J. Chirravuri, and W. J. Miniscalco
}

\begin{abstract}
We have experimentally and theoretically investigated the effects of detuning the pump wavelength on the gain and noise properties of small-signal, erbium-doped fiber amplifiers co-directionally pumped in the $980-\mathrm{nm}$ band. While the pump wavelength can be varied over a wide range with little impact on the gain, a noise penalty is incurred. For amplifiers saturated by amplified spontaneous emission, it is possible to increase the gain by detuning the pump wavelength.
\end{abstract}

\section{INTRODUCTION}

$\mathrm{T}$ HE 980-nm pump band is the most efficient for erbiumdoped fiber amplifiers (EDFA's). It is relatively narrow, however, and the limits this imposes on pump laser wavelength is a source of concern [1], [2]. Recently, it has been demonstrated that the restrictions on pump wavelength are less severe than had been originally believed, and it is possible to obtain relatively constant gain over a broadpump-wavelength range for both small-signal [3] and power [4] amplifiers. In this work, we examine in detail the effects of pump-wavelength detuning on small-signal EDFA's, including the first measurements of noise penalties. Theoretical and experimental investigations reveal relaxed pump-wavelength requirements with respect to gain, but also a noise figure penalty that increases $0.6 \mathrm{~dB}$ for each $10 \mathrm{~nm}$ of detuning. In addition, we report the observation that the performance of EDFA's saturated by amplified spontaneous emission (ASE) can actually be improved by pump-wavelength detuning. This results from a reduction of the amount of pump power that is lost to backward ASE. We conclude that for applications in which noise figure is not paramount it is not necessary to use pump lasers close to the peak of the absorption band.

\section{Small-Signal Gain and NoIse Figure}

In the experiments pumping was provided by the output of a Ti:sapphire laser that was combined with the 1551-nm signal by a WDM coupler. The gain, $G$, was measured using a lock-in amplifier to separate the signal from the spontaneous emission. Determining the noise figure also requires a measurement of the ASE power. This was accomplished using a bandpass filter ( $F W H M \approx 1.8 \mathrm{~nm}$ ) centered on the signal wavelength. The transmitted power was measured with

Manuscript received December 27, 1991; revised March 30, 1992.

B. Pedersen is with the Electromagnetics Institute, Technical University of Denmark, DK-2800 Lyngby, Denmark.

J. Chirravuri and W. J. Miniscalco are with GTE Laboratories Inc., Waltham, MA 02254.

IEEE Log Number 9200929. the signal turned off and divided by the effective bandwidth of the filter to obtain the forward ASE power spectral density, $S_{\mathrm{ASE}}\left(\nu_{s}\right)$, at the signal frequency, $\nu_{s}$, exiting the EDFA. The noise figure was evaluated using the expression $F=$ $\left[S_{\mathrm{ASE}}\left(\nu_{s}\right) / h \nu_{s}+1\right] / \mathrm{G}$.

Fig. 1 plots (a) the small-signal gain and (b) the noise figure as functions of the launched pump power for different pump wavelengths. The $\mathrm{Ge} / \mathrm{Al} / \mathrm{P} /$ Er-doped fiber had an NA of 0.18 and cutoff of $940 \mathrm{~nm}$. Signal powers were low enough not to affect the gain. The measured values are indicated by marks and the solid curves are simulations of the experiment performed using a comprehensive numerical model [5] for which all input parameters, including erbiumand refractive-index profiles, have been experimentally determined. The fiber length is $35 \mathrm{~m}$, which is the optimum length for $120 \mathrm{~mW}$ of pump power at $979 \mathrm{~nm}$, i.e., any change in the fiber length will decrease the gain. The gains for the other pump wavelengths, however, can still be improved by increasing the fiber length. A somewhat surprising result is that both theory and experiment indicate that the gain for 969 and $959 \mathrm{~nm}$ is higher than that for $979 \mathrm{~nm}$, the wavelength with the highest absorption cross section. Lower gains were previously reported pumping at $979 \mathrm{~nm}$, but in that instance it was attributed to using fiber lengths that were longer than optimum [3]. We have demonstrated here that it is possible to increase the gain by detuning the pump wavelength, even for amplifiers with optimum length. This effect results from the high level of ASE in the fiber and will be explored in the next section.

In contrast, Fig. 1(b) indicates that the noise figure can only be degraded by detuning, for these conditions increasing by $\approx 0.6 \mathrm{~dB}$ for each $10 \mathrm{~nm}$ the pump wavelength differs from $979 \mathrm{~nm}$. In general, a good noise figure requires that a high inversion be maintained at the input end of the fiber over roughly the length required to provide the first $10-15 \mathrm{~dB}$ of signal gain. For a highly driven small-signal amplifier the major perturbation is the backward ASE, and obtaining a high inversion in this region requires that the pump rate be large compared to stimulated emission rate due to backward ASE. Since the pump rate is proportional to the pump absorption cross section, the best noise performance is always obtained at the peak of the absorption band.

\section{Improvement of Gain by Pump Detuning}

Fig. 2 plots the calculated small-signal gain as function of pump wavelength for three pump powers and the fiber used 

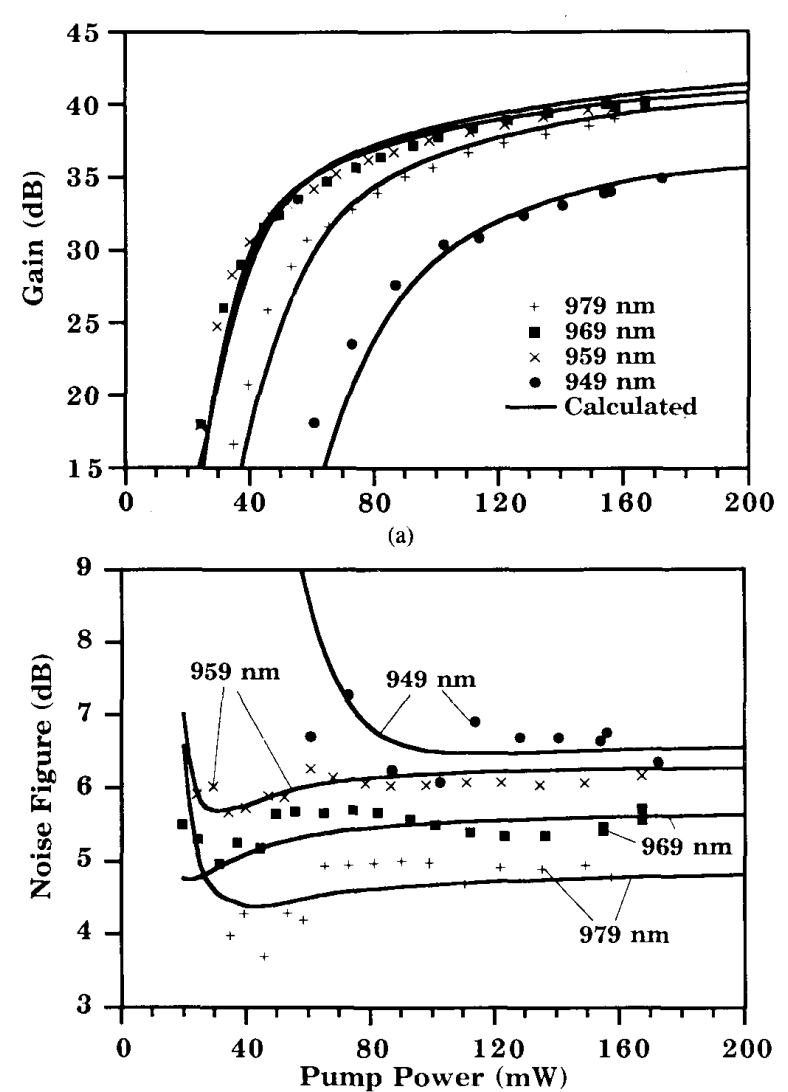

(b)

Fig. 1. (a) Gain and (b) noise figure as a function of pump power for the pump wavelengths indicated.

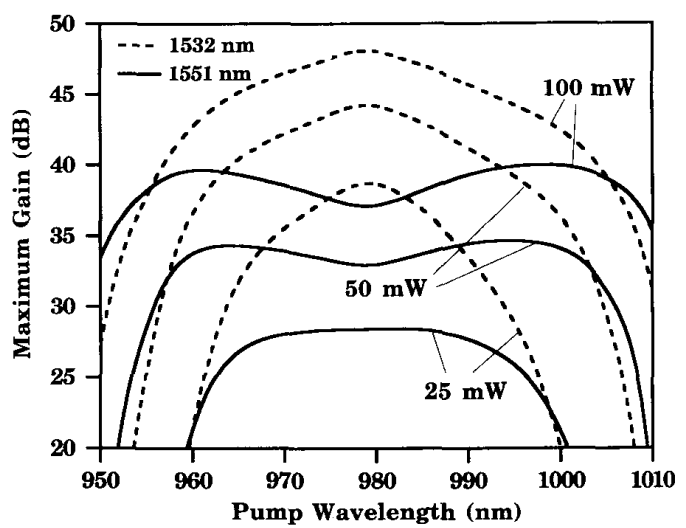

Fig. 2. Maximum gain versus pump wavelength for the signal wavelengths and pump powers indicated. Fiber lengths have been adjusted to maximize gain.

in Fig. 1. The signal wavelengths are 1532 and $1551 \mathrm{~nm}$, and the fiber lengths are those giving the highest gain. The gain is seen to be extremely tolerant to pump wavelength: for pump powers of 25,50 , and $100 \mathrm{~mW}$, the $3-\mathrm{dB}$ bandwidths are $16.5,22.5$, and $27 \mathrm{~nm}$ for a signal at $1532 \mathrm{~nm}$, and $33,47.5$ and $56 \mathrm{~nm}$ for one at $1551 \mathrm{~nm}$. The wider bandwidths for
$1551 \mathrm{~nm}$ arise from a more favorable emission to absorption cross section ratio which permits gain with lower excited state populations. Fig. 2 indicates that for a signal at the peak of the stimulated-emission cross-section spectrum $(1532 \mathrm{~nm}$ for the present fiber), the optimum pump wavelength will always be at the peak of the pump absorption $(979 \mathrm{~nm})$ as expected. For a signal in the shoulder of the emission spectrum $(1551 \mathrm{~nm})$, however, it is possible to obtain higher gain by detuning the pump wavelength $\approx 20 \mathrm{~nm}$.

The explanation for this detuning enhancement is provided by Fig. 3 which plots the gain together with the pump, forward and backward ASE powers as functions of the location in the fiber for two pump wavelengths. The signal wavelength is $1551 \mathrm{~nm}$, the pump power is $100 \mathrm{~mW}$, and the fiber lengths are chosen for maximum gain: $32 \mathrm{~m}$ for $979 \mathrm{~nm}$ and $46 \mathrm{~m}$ for $999 \mathrm{~nm}$. The fiber can be divided into three sections whose approximate boundaries are indicated in Fig. 3 by changes in slope for the gain and pump power. In the first section both the pump and the backward ASE are strong, and this combination leads to significant recycling of the erbium ions through stimulated emission and pump absorption. Consequently, there is a significant transfer of power from the pump to the backward ASE as indicated by the steep slopes for both curves. In the middle section both the forward and the backward ASE powers are low and the inversion is high since the only de-excitation process is spontaneous emission. In this region the gain increases most rapidly and the pump attenuation is lowest. In the final section the forward ASE has reached a level where stimulated emission has again become important but the low pump intensity is not sufficient to maintain inversion and the gain per unit length falls off.

Pumping at $999 \mathrm{~nm}$ provides higher gain than pumping at $979 \mathrm{~nm}$ because less pump power is lost to backward ASE in the first section of the fiber. This occurs since the lower absorption cross section at $999 \mathrm{~nm}$ reduces the rate at which erbium ions are re-excited and then de-excited by stimulated emission into the backward ASE. This can be seen by examining the point in Fig. 3 where the backward ASE is equal to $0 \mathrm{dBm}$, roughly the location of the boundary between the first and second region. Although the gain is the same for both the pump wavelengths, roughly twice as much pump power remains at $999 \mathrm{~nm}$ than at $979 \mathrm{~nm}$. In effect, pump power is transferred to the second region of the fiber where it is more efficient at producing gain. This is consistent with the higher noise figure of the 999-nm-pumped amplifier: the lower pump rate which reduces loss to backward ASE also means a lower population inversion in the critical first section of the amplifier.

In addition to providing useful insights into the complex interactions occurring within an EDFA, the above investigations can provide information of practical importance. Since a major factor in the cost of diode pump lasers is the low yield for wavelengths near $979 \mathrm{~nm}$, the relaxed tolerances seen in Fig. 2 could lead to a significant reduction in EDFA cost. It may also be possible to exploit the gain improvement due to detuning seen in Fig. 2. Fig. 4 plots (a) the maximum gain for $979 \mathrm{~nm}$ pumping, (b) the maximum gain improve- 

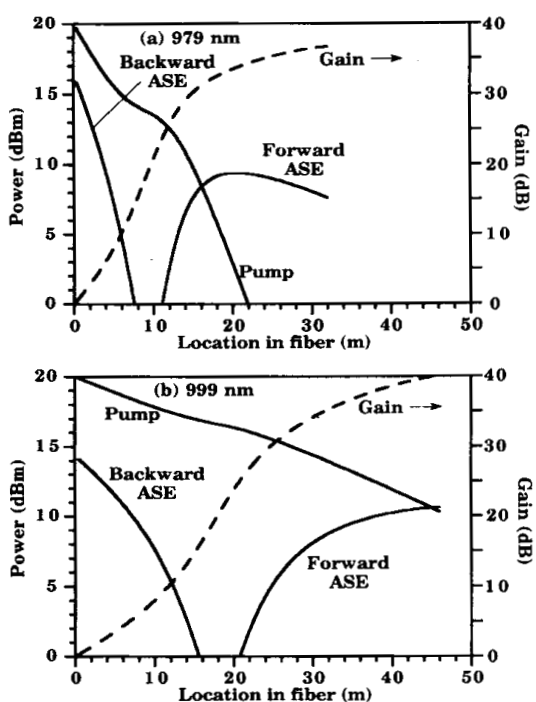

Fig. 3. Pump power, ASE power, and signal gain as a function of position within fiber for pump wavelengths of (a) $979 \mathrm{~nm}$ and (b) $999 \mathrm{~nm}$

ment from detuning, and (c) the optimum amount of detuning, all as functions of the pump power. Comparing Figs. 4(a) and (b) confirms that only for amplifiers saturated by ASE $(G>25 \mathrm{~dB})$ is it possible to obtain a gain improvement by detuning the pump wavelength. Fig. $4(\mathrm{~b})$ indicates that while the pump power at which the improvement first appears decreases with increasing NA, a greater benefit is obtained for fibers with low NA's. Fig. 4(c) shows that the amount of detuning for greatest improvement initially depends on pump power but eventually saturates at values in the 20-24 nm range. This detuning-induced gain enhancement can provide a significant improvement. For example, using an NA of 0.25 the maximum gain is $33.8 \mathrm{~dB}$ at $1551 \mathrm{~nm}$ when pumping with $50 \mathrm{~mW}$ at $979 \mathrm{~nm}$. However, the same gain can be obtained with only $30 \mathrm{~mW}$ by detuning the pump wavelength $\approx 20 \mathrm{~nm}$.

\section{Conclusions}

Experimental and theoretical investigations of 980-nmpumped small-signal EDFA's have revealed significantly relaxed tolerances on pump wavelength if gain is the primary consideration. This is expected to have an important impact on the yield and cost of diode lasers for this pump band. A noise figure penalty of $0.6 \mathrm{~dB}$ per $10 \mathrm{~nm}$ of detuning is incurred, however, and must be considered in preamplifier applications. For ASE-saturated EDFA's near $1550 \mathrm{~nm}$, it is possible to obtain a useful improvement in efficiency by detuning since this reduces the pump power lost to backward ASE.

\section{ACKNOWLEDGMENT}

We wish to thank M. Dakss, T. Wei, and B. A. Thompson for experimental assistance, and L. Andrews and R. Lauer for helpful discussions and suggestions.

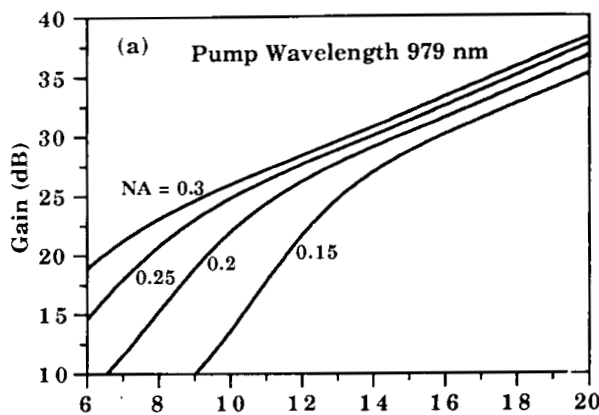

(a)
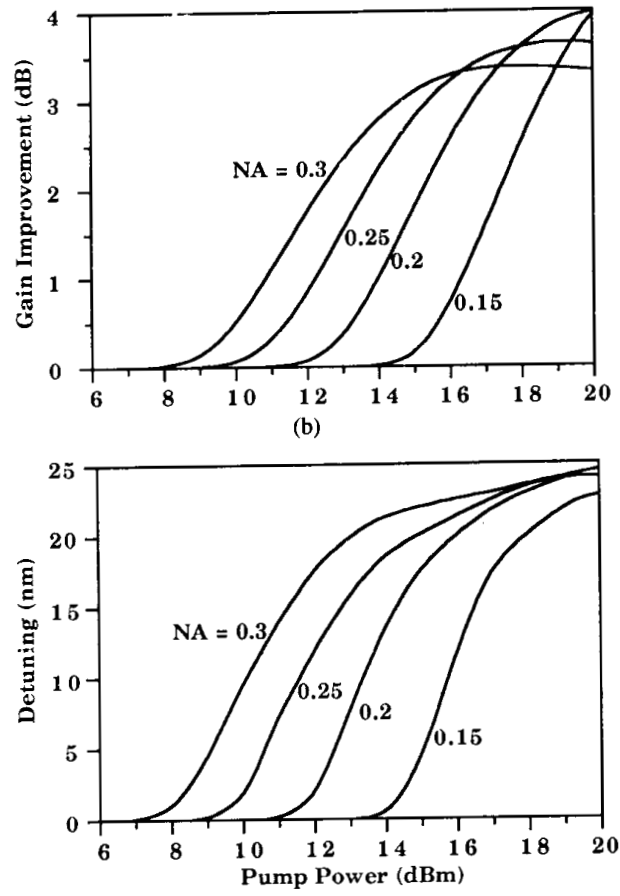

(c)

Fig. 4. (a) Gain for 979-nm pump wavelength, (b) gain improvement due to detuning, and (c) amount of detuning for maximum gain improvement. Calculations used step-index fibers with $850-\mathrm{nm}$ cutoff wavelength. Lengths that maximized gain were used

\section{REFERENCES}

[1] P. C. Becker, A. Lidgard, J. R. Simpson, and N. A. Olsson, "Erbium-doped fiber amplifier pumped in the $950-1000 \mathrm{~nm}$ region," IEEE Photon. Technol. Lett., vol. 2, pp. 35-37, 1990.

[2] M. Wada, K. Yoshino, M. Yamada, and J. Temmyo, "0.98-mm strained quantum well lasers for coupling high optical power into single-mode fiber," IEEE Photon. Technol. Lett., vol. 3, pp. 953-955, 1991.

[3] R. M. Percival, S. Cole, D. M. Cooper, S. P. Craig-Ryan, A. D. Ellis, C. J. Rowe, and W. A. Stallard, "Erbium-doped fibre amplifier with constant gain for pump wavelengths between 966 and $1004 \mathrm{~nm}$,' Electron. Lett., vol. 27, pp. 1266-1268, 1991.

[4] B. Pedersen, J. Chirravuri, and W. J. Miniscalco, "Gain and noise penalty for detuned $980-\mathrm{nm}$ pumping of erbium-doped fiber power amplifiers," IEEE Photon. Technol. Lett., vol. 4, pp. 351-353, 1992.

[5] B. Pedersen, A. Bjarklev, J. Hedegaard Povlsen, K. Dybdal, and C C. Larsen, "The design of erbium-doped fiber amplifiers," J. Lightwave Technol., vol. 9, pp. 1105-1112, 1991. 